\title{
Design and Manufacturing of Motor-Tendon Actuator for a Soft Starfish-Like Robot
}

\author{
M. Munadi*, Mochammad Ariyanto, \\ Joga D. Setiawan, Edo Purwanto, \\ Taufiqurohman ${ }^{5}$ \\ Dept. of Mechanical Engineering, \\ Diponegoro University \\ Semarang, Indonesia \\ *munadi@undip.ac.id
}

\author{
M. Amirullah Akbar, A. Hasan Fauzi \\ Garuda Maintenance Facility AeroAsia, \\ Soekarno Hatta Intern. Airport, \\ Cengkareng, Indonesia
}

\author{
Muhammad Nanda Setiawan \\ Dept. of Physic Energy Engineering, \\ Surya University, \\ Tangerang, Indonesia
}

\begin{abstract}
A motor-tendon actuator is one of the widely used actuators in a soft robot that utilizes rope/wire connected to a motor. Soft tendon actuator is an alternative solution to the shortcomings of several other types of actuators such as pneumatic-network actuators, and shape memory alloy (SMA) actuators. This study presents the design process and manufacturing of motor-tendon actuators that can be used as an actuator for a starfish-like robot. Five soft motor-tendon actuators will be developed by varying the dimensions and composition of silicone rubber material. In the manufacturing process, a casting method is used to build four soft legs of the starfish-like robot. The tests for actuator bending are performed on the five developed motor-tendon actuators. Based on the test results, the bending angle of the proposed actuator is affected by the dimension of the soft leg, while the flexibility of the actuator is influenced by tendon thickness. Based on the composition of the soft leg material, the smaller the ratio of the composition of silicon rubber to the catalyst, the shorter the pot life material, and the faster curing time.
\end{abstract}

Keywords- motor-tendon actuator, soft robot, silicone rubber, pot life, curing time

\section{INTRODUCTION}

The development of the quality of robotic materials always increases every year. Nowadays, robots are designed to be able to adapt to the track in accordance with human expectations. More and more robots are involved in daily human life both in terms of work and other activities. As we know, among the types of robots that are growing rapidly are hard robots. Even though in the last 50 years, hard robots have always been growing rapidly, they still have weaknesses [1]. Hard robots are comprised of rigid mechanical structures such as metals or plastics. Hard robots are usually bulky and have limited motion.

In order to overcome the weaknesses of the hard robots mentioned above, some researchers are inspired by the biological structure found in animals such as octopus tentacles, elephant trunks, etc. To mimic this biological structure, researcher around the world start designing and building soft robots [2]. Hard robots are more like machines than soft robots. With infinite soft structure and degrees of freedom (DoF), this type of robot can be used for monitoring tasks in an irregular or unstructured environment. This robot became known as a soft robot which became a new classification in the world of robotics. Soft robots are in great demand because they have the ability to adapt to the environment irregularly [3, 4].

There are several robot components that play an important role in both hard robots and soft robots. One of the most important components is the utilized actuator. An actuator is needed to drive the robot. A motor is widely used actuator in the hard robot. Many types of actuators are employed in soft robots such as Shape Memory Alloy (SMA) actuators [5], pneumatic network (pneu-net) actuators [6], and motor-tendon actuators. However, this type of actuator has limitations. For example, pneu-net actuators have deficiencies on the surface where the material is prone to tear. By tearing the surface of the pneu-nets actuator, all parts of the actuator will be damaged. As a result, this type of actuator is very vulnerable to use in areas that have a rough and sharp surface. This type of actuator has certain requirements in its use [7, 8]. While the SMA actuator is quite difficult to manufacture compared to pneu-net actuator. Motor-tendon actuator is relatively easier to manufacture compared to SMA and pneu-net actuator. The mass of this actuation system can be lightweight enough [9].

This paper proposes the design and manufacture of motor-tendon actuator used in a starfish-like robot. Five motor-tendon actuators will be built by varying the dimensions and composition of silicone rubber material. The developed actuators are tested in term of flexibility and formed bending angle.

\section{SOFT STARFISH-LIKE ROBOT}

Starfish are radial symmetry animals and generally have four or more arms as a walking tool. Because of its ability to run in all terrain and trajectories that do not have good structure, researchers are interested in developing soft starfish-like robot from starfish animals. The soft starfishlike robot design is shown in Figure 1. Soft starfish-like robots that are designed have four arms.
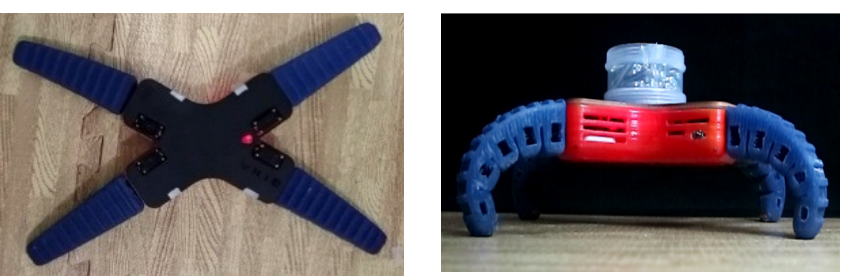

Fig. 1. Soft starfish-like robot.

To develop a soft starfish-like robot, we try to develop low-cost soft motor-tendons actuators that are used as a component of a robot actuator. Actuator motors are selected because this actuation system has a compact system and provides more force.

\section{PROPOSED MOTOR-TENDON ACTUATOR}

The soft motor-tendon actuator is one of the soft actuators that utilize rope/wire as a tendon connected to the 
servo motor arm. The advantage of the proposed motortendon actuator is that it can adapt to irregular terrain or trajectories, for example, the robot can walk on sharp surfaces such as small and pointed gravel.

\section{A. Actuator Design}

In this study, the first stage was to design the soft arm model of the motor-tendon actuator so that a technical image was produced. The soft tendon actuator was designed using 3D CAD software (SolidWorks). The soft and minimalist proportional 3D design of the proposed motortendon actuator is depicted in Figure 2.

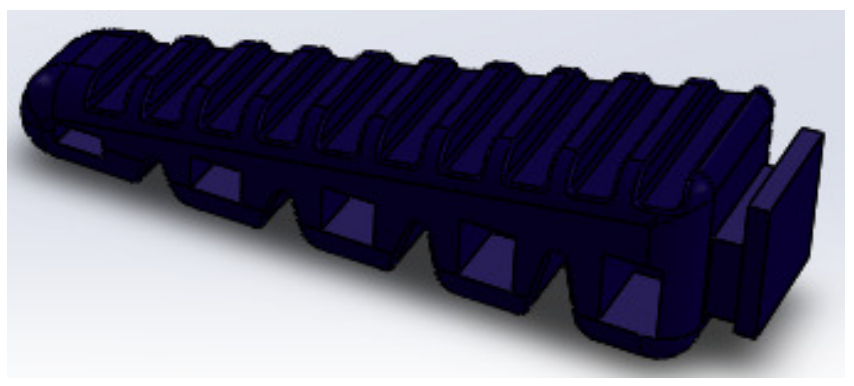

Fig. 2. Soft arm actuator design

\section{B. Motor-Tendon Actuator Manufacture}

The soft motor-tendon actuator is expected to be able to run on terrain or irregular trajectories. It must match for some of the criteria that we specify. Among these criteria is tendon elasticity. In order to fulfill the criteria of tendon elasticity, silicone rubber is chosen as a solution for the type of material. Silicone rubber is a type of synthetic polymer that has the advantage of weather resistance, aging, resistance to heat temperature up to $250^{\circ} \mathrm{C}$. Silicon rubber is stable over a wide enough temperature range from $100^{\circ}$ to $250^{\circ} \mathrm{C}$. When compared to natural rubber, silicone rubber has better fire resistance properties and electrical insulation. Properties possessed such as volume resistance, dielectric strength, and other strength factors that are not affected by temperature changes are referred to as thermal stability. In addition to several advantages as mentioned above, silicone rubber has a unique on the surface that is anti-sticky. This makes it much needed for intermediaries or as a means or layer to prevent bonding or sticking to other objects.

In the manufacturing process using silicone rubber, there are four methods of formation, including extrusion, calendaring, coating, and molding and casting [10]. For complex shapes, more casting methods are chosen. The casting method is a conventional method used in the manufacturing process by using mold, where the liquid material is inserted into it and will compact according to the shape of the mold [11]. This method is a method that requires several steps to complete it. In the casting process, a mold is needed as a material container for the product so that the planned soft motor- tendon actuator is produced using 3D printers.

\section{Actuator Casting}

The manufacturing process of soft motor-tendon actuators using the casting method consists of several stages, including mold design, mold molding, material preparation, and vacuum material, pouring, de-molding, and finishing. The following is an explanation of each of these stages.

\section{- Mold design}

In the manufacturing process of soft motor-tendon actuators, a mold is needed as a mold container. The mold design adapts to the desired soft actuator design. Figure 3 shows the mold design for the soft actuator that will be utilized on the starfish-like robot.
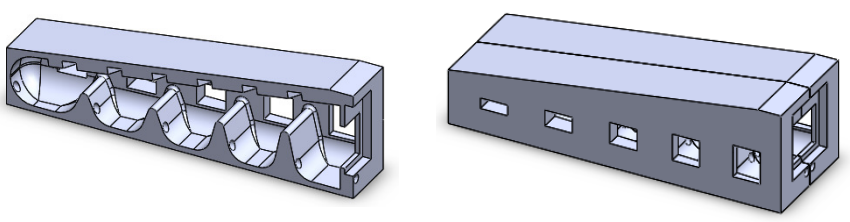

Fig. 3. Mold design for the soft actuator

\section{- Mold print}

Generally, the molding process is performed using sand and metal. This simple method is quite easy to carry out. However, molds printed in this way have disadvantages, namely the difficulty of changing mold print variations. The solution is to print molds with various variations, without the need to print the reference form first is to use a $3 \mathrm{D}$ printer. The process of producing molds using 3D Printer has the advantage of being able to adjust to the complex shape and high level of accuracy. Therefore, the process of printing mold print in this study uses a 3D printer where the material comes from polylactic acid (PLA) as shown in Figure 4.

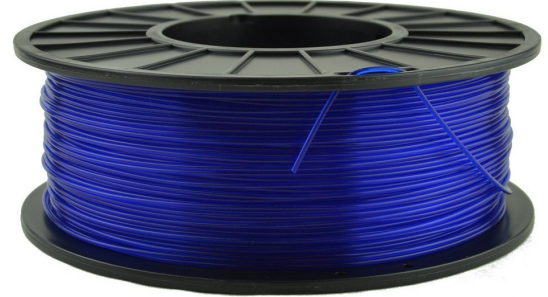

Fig. 4. Polylactic acid (PLA)

\section{- Material preparation}

The type of silicone rubber used is RTV-52 (room temperature vulcanizing) silicone. This material has a texture that is not too soft and not too stiff, so it is suitable for use as a material for manufacturing soft motor-tendon actuator. Silicone rubber RTV 52 consists of two compositions, namely rubber, and catalyst. The percentage of the standard composition used is $40: 1$, meaning that when using $40 \mathrm{gr}$ rubber then the catalyst that must be added is 1 gr. In this study, the composition of rubber and catalysts will be varied to obtain the best quality and suitable for soft starfish-like robot applications.

\section{- Vacuum Material}

When silicone rubber is mixed with a catalyst, air bubbles will form. This air bubble will cause porosity. This porosity can be seen when the casting results have become the final product. Therefore, the vacuum machine is used to remove air bubbles when the silicone rubber is mixed with the catalyst [8]. The used vacuum in this research is shown in Figure 5. 


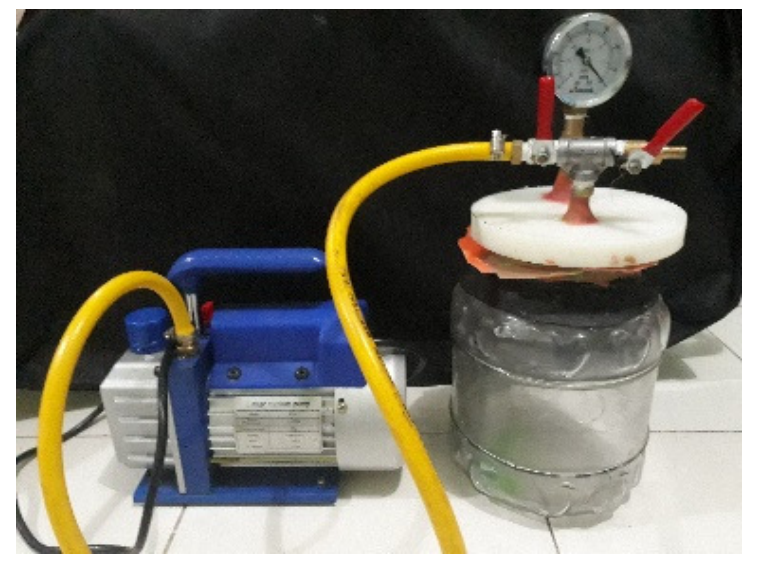

Fig. 5. Vacuum material

\section{- Pouring}

The next stage after the material is ready, pouring is performed which is the technique of pouring silicon rubber into the mold. Pouring silicon rubber into the mold should not be too fast, nor can be too slow. This will affect the air trapped in the mold and will produce bubble cavities when the silicon rubber has hardened. The mold is then stored in the room at room temperature, because the RTV silicone rubber will solidify itself at room temperature according to its curing time of \pm 6 hours.

\section{- Demoulding and Finishing}

After silicon rubber hardens, the results of silicon rubber can be taken from the mold, called the de-molding process. The last step is finishing which is carried out to smooth the casting results so that the soft tendon actuator is in accordance with the initial design. Usually, it is made by cutting the parts that are less neat on the ends of the product.

\section{RESULTS AND DISCUSSION}

In this study, soft actuator tendons were produced by varying five soft actuators based on dimensions to obtain the best design for a starfish-like robot. Figure 6 shows the shape of the soft design of the motor-tendon actuator. From the design, several dimensions were changed based on experimental needs. Table I shows the dimensions of the five developed soft motor-tendon actuators.

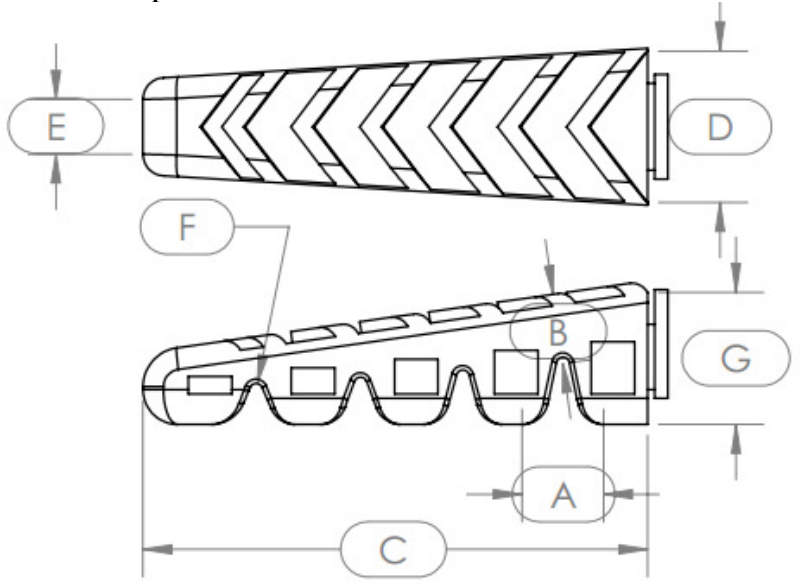

Fig. 6. The model of soft tendon actuator
TABLE I. THE DIMENSION OF THE SOFT ACTUATORS

\begin{tabular}{|c|c|c|c|c|c|c|c|}
\hline $\begin{array}{c}\text { Motor- } \\
\text { Tendon } \\
\text { sample }\end{array}$ & $\begin{array}{c}\mathbf{A} \\
(\mathbf{m m})\end{array}$ & $\begin{array}{c}\mathbf{B} \\
(\mathbf{m m})\end{array}$ & $\begin{array}{c}\mathbf{C} \\
(\mathbf{m m})\end{array}$ & $\begin{array}{c}\mathbf{D} \\
(\mathbf{m m})\end{array}$ & $\begin{array}{c}\mathbf{E} \\
(\mathbf{m m})\end{array}$ & $\begin{array}{c}\mathbf{F} \\
(\mathbf{m m})\end{array}$ & $\begin{array}{c}\mathbf{G} \\
(\mathbf{m m})\end{array}$ \\
\hline Tendon 1 & 16.87 & 12.77 & 120 & 30 & 16.16 & 3 & 33 \\
\hline Tendon 2 & 19.67 & 11.67 & 115 & 36 & 18.24 & 3 & 31.85 \\
\hline Tendon 3 & 19.94 & 9.53 & 100 & 30 & 18.10 & 1.5 & 30.98 \\
\hline Tendon 4 & 21.45 & 16.37 & 125.8 & 39 & 25 & 3 & 30.98 \\
\hline Tendon 5 & 18.72 & 16.10 & 116 & 34.8 & 12.59 & 1.5 & 30.37 \\
\hline
\end{tabular}

Table I shows a description of each dimension for each soft actuator. Based on the dimensions of the soft model of the actuator tendon, a mold is produced as an actuator mold container. Mold is printed using 3D printer made from filament polylactide acid (PLA). Figure 7 shows the type of mold used.

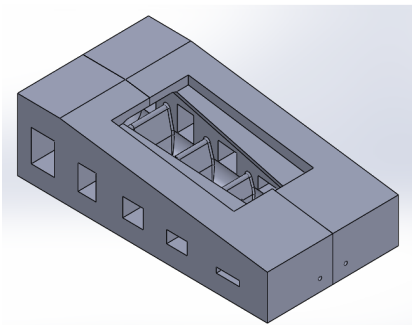

(a)

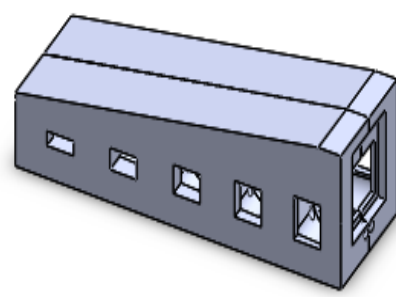

(b)
Based on Figure 7, the difference between mold type 1 and mold type 2 lies in the position of the casting hole. This difference is a problem that needs to be considered in the manufacturing of soft actuator. Type 1 mold has advantages in the pouring process where the material is easier to pour even though the pot life of the material is faster than the standard usage. However, the printout needs more finishing, and the castings are too large, so they are prone to spillage. While type 2 mold has the advantage of being safe and stable at curing time. In the curing time, the stability of the mold needs to be maintained so that the results are maximum. Disturbances during curing time include many things such as shifting mold, dust, small objects around the mold and so on. While the disadvantage of type 2 mold is the process of pouring material into a mold that takes a long time. Furthermore, Figure 8 reveals the results of the soft casting of the soft motor-tendon actuators.

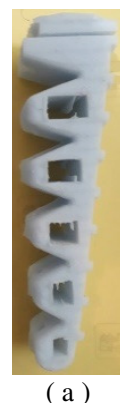

( a )

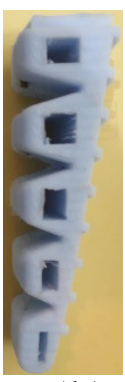

( b )

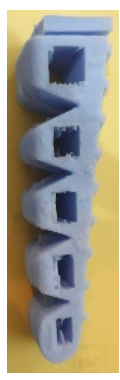

( c )

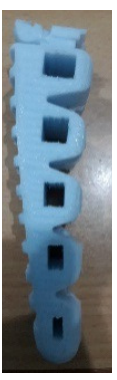

(d)

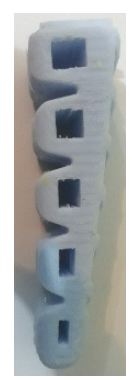

( e )
Fig. 8. The results of the casting sample for the soft motor-tendon actuator.

Based on the results from the obtained casting, there are several parameters that need to be considered. Among them are formed angles or bending angles, thickness, pot life, and curing time of the soft actuator tendon. 
Bending test is performed to find out the ability of each soft motor-tendon actuator to bend. The bending test is conducted manually using a servo motor and microcontroller circuit as a test tool. Figure 9 shows the bending test results of the five soft motor-tendon actuators. The bending test is performed by giving the servo motor arm a command to vary the arm angle $30^{\circ}, 60^{\circ}$, and $90^{\circ}$. Based on the bending test, the results are that the angles formed at each tendon are different. This is caused by several factors, including the length of the tendon (value $\mathrm{C}$ ); distance $\mathrm{B}$; and distance $\mathrm{A}$ (Table I). Tendon 1 test produces angles of $10^{\circ}, 30^{\circ}$ and $45^{\circ}$. These results show a high error value, which reached $50 \%$. Based on the results of the tendon 1 bending test, it is stated that the bending angle does not match the one formed.

Tendon 2 test produces angles of $25^{\circ}, 50^{\circ}$, and $60^{\circ}$. The error value of tendon 2 is at an angle of $30^{\circ}$ and $60^{\circ}$ at $20 \%$. While the test at $90^{\circ}$ experienced an error of $50 \%$. Furthermore, testing is carried out on tendon 3. Testing of tendon 3 produced angles of $50^{\circ}, 80^{\circ}$, and $90^{\circ}$. The tendon error value 3 at an angle of $50^{\circ}$ and $80^{\circ}$ are $40 \%$ and $25 \%$, respectively. Whereas in tendon 3 the formation of a perfect angle is at an angle of $90^{\circ}$. But at $90^{\circ}$ angle, the result of bending is too bent inward, consequently, if the tensile angle is enlarged again the bending that occurs in the tendon will be too large.

Tendon 4 test produces angles of $50^{\circ}, 70^{\circ}$, and $90^{\circ}$. Tendon error values 4 at angles $50^{\circ}$ and $70^{\circ}$ are $40 \%$ and $14.2 \%$, respectively. Like tendon 3 , tendon 4 also undergoes perfect angle formation at an angle of $90^{\circ}$. Further testing of tendon 5 produces angles of $45^{\circ}, 75^{\circ}$, and $90^{\circ}$. Tendon error values 5 at angles of $45^{\circ}$ and $75^{\circ}$ are $33.3 \%$ and $20 \%$, respectively. As with tendons 3 and 4, tendon 5 also undergoes perfect angle formation at an angle of $90^{\circ}$. So tendons 3 have the smallest angle error.

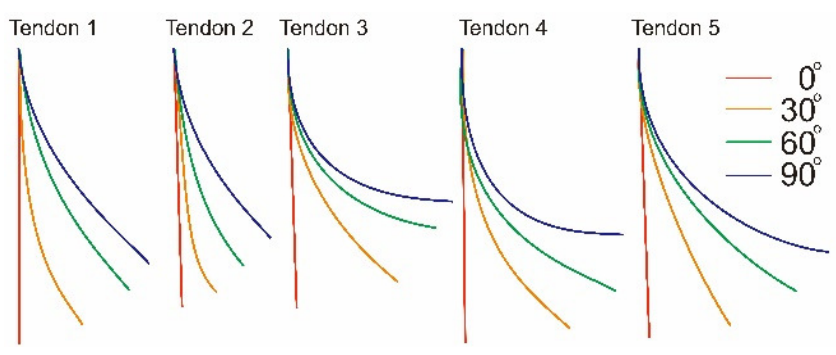

Fig. 9. Banding angle test

To strengthen the contraction test results of $30^{\circ}, 60^{\circ}$ and $90^{\circ}$ angles, the researchers also conducted the maximum angle test formed on each tendon and shown in Figure 10. The maximum angle is limited by the servo motor power, in which it is conducted by giving a pull until the tendon reaches the highest angle of the limit to be able to be pulled. Tendons $1,2,3,4$, and 5 each reach the angles of $173^{\circ}$, $152^{\circ}, 124^{\circ}, 133^{\circ}$ and $133^{\circ}$. The maximum angle formed in each tendon is not recommended for use in a robot's operation or other uses because we consider being the critical angle of the tendon work area. For example, when the soft actuator will be used on a soft starfish-like robot with a maximum angle of $90^{\circ}$.

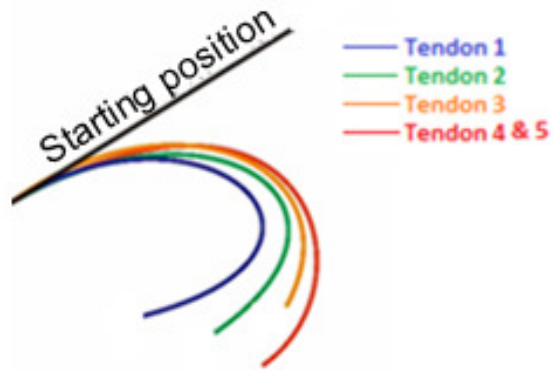

Fig. 10. The maximum bending angle of five developed motor tendons.

Based on Figure 11, tendon 1 with value A of $16.87 \mathrm{~mm}$ and $\mathrm{C}$ of $120 \mathrm{~mm}$ will produce a maximum capable angle of the shape of $173^{\circ}$. Tendon 2 with value $\mathrm{A}$ of $19.67 \mathrm{~mm}$ and $\mathrm{C}$ of $115 \mathrm{~mm}$ will produce a maximum angle of $152^{\circ}$. Tendon 3 with value A amounting to $19.94 \mathrm{~mm}$ and $\mathrm{C}$ of $100 \mathrm{~mm}$ produces a maximum angle of $124^{\circ}$. Tendon 4 with value $\mathrm{A}$ of $21.45 \mathrm{~mm}$ and $\mathrm{C}$ of $125.8 \mathrm{~mm}$ produces a maximum angle of $133^{\circ}$. Tendon 5 with value $\mathrm{A}$ of 18.72 $\mathrm{mm}$ and $\mathrm{C}$ of $116 \mathrm{~mm}$ produces a maximum angle of $133^{\circ}$. These results, then the tendon will have a large maximum angle value with the requirements, the greater the value of parameters $\mathrm{A}$ and $\mathrm{C}$ (Table I). Vice versa, if the value of parameters $\mathrm{A}$ and $\mathrm{C}$ gets smaller, the maximum angle formed on the tendon also gets smaller.

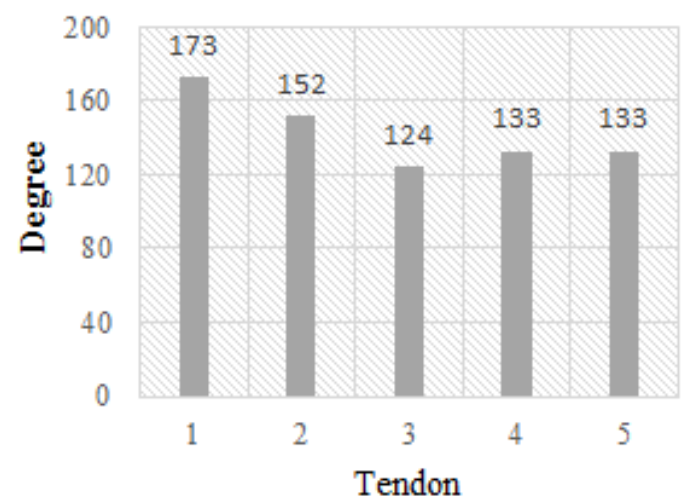

Fig. 11. The relationship of values $\mathrm{A}$ and $\mathrm{C}$ to the maximum bending angle

In addition to the bending test, an elasticity of the soft actuator test is also carried out when it is stationary. This is performed to find out how flexible the tendon is when it is without load and without tensile forces. Figure 12 shows the results of the soft elasticity of the actuator tendon. For tendon 1 , the angle formed is $75^{\circ}$, the angle on the tendon 2 is $65^{\circ}$, the angle on the tendon 3 is $50^{\circ}$, and the angle on the tendons 4 and 5 is $60^{\circ}$. This angle indicates the strength of the tendon in holding its load. The greater the angle formed means the smaller the strength of the tendon in holding the load or the higher the flexibility. This also means the lower the strength of the tendon to return to its original shape if bending occurs when given the force of attraction. Based on the fifth flexibility test of the tendon model, tendon 1 has the highest flexibility compared to the other models, and tendon 3 has the lowest flexibility compared to other tendon models. In other words, the parameter that greatly affects the degree of tendon flexibility is the thickness or B value in Table I. 


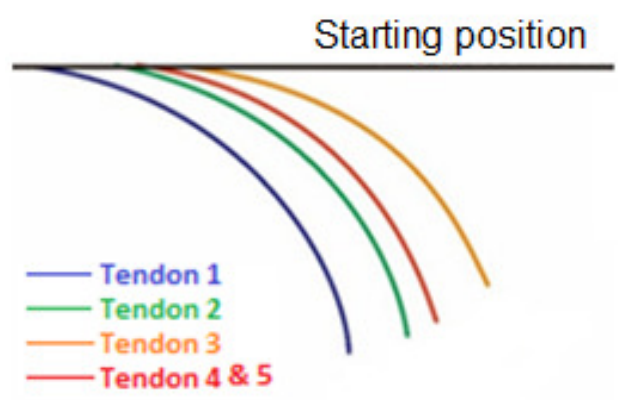

Fig. 12. Tendon flexibility without load and without tensile force

The performances of the soft actuator produced are not only influenced by dimensions but also other things that affect the ability of the soft actuator are the result of casting. The casting results are strongly influenced by the composition of the material used in making soft actuator. In this study, the material used is silicone rubber type RTV-52. In its use, RTV -52 requires a catalyst as a hardener. The composition of RTV-52 and catalyst with a ratio of $35: 1$, 25:1, and 20:1. After being mixed between RTV-52 and the catalyst, this material has a very short pot life. Pot life is the time needed to work by silicone rubber before it is poured in the mold.

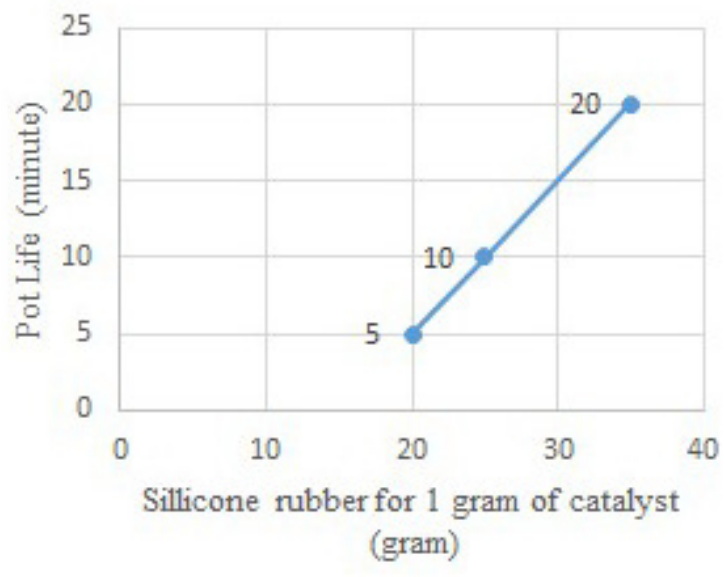

Fig. 13. Comparison of material composition to pot life

Figure 13 shows a comparison between the compositions of the soft tendon actuator material for pot life. Based on Figure 13, it is known that the smaller the comparison of silicon rubber composition with the catalyst, the shorter the pot life material. This has an impact on the process of pouring material into the mold. If the pot life is short, a short time is needed for the pouring process. In short, the pouring process into the mold resulted in the material not filling the mold in full. This will affect the results of casting, where if the material does not fill fully the mold then there are some porosities. In fact, if the pot life is too short, it will produce many porosities to damage the casting results.

Furthermore, Figure 14 shows a comparison of material composition to curing time. Curing time is the time needed by the silicone rubber to be poured perfectly. From the results of the curing time, it is known that with a composition between RTV-52 and a catalyst for a ratio of $20: 1$, the curing time is around 6 hours. With a composition between RTV-52 and a catalyst for a ratio of 25:1, curing time is around 8 hours. While the composition of the RTV52 material and catalyst of 35:1 requires curing time to be 10 hours. Based on the results of the curing time test, it can be seen that the greater the composition of the silicone rubber to the catalyst, the longer the curing time. Vice versa, with the comparison of the composition of the silicone rubber material getting smaller with the catalyst, it will result in faster curing time.

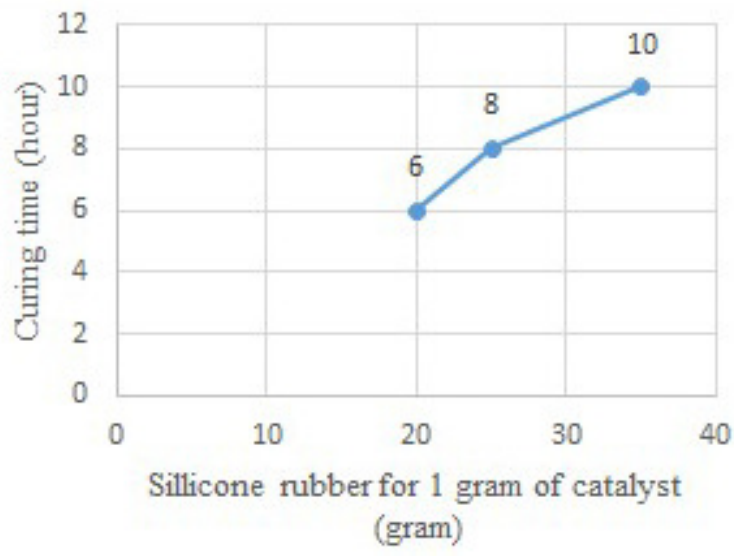

Fig. 14. Comparison of material composition to curing time

This study also conducted a hardness test on the material of the soft actuator using a type A durometer that has force of 821 gf. Type A durometers measure the hardness of neoprene, EPDM rubber, polyurethane, printing rollers, silicone, white PVC and other similar materials. Figure 15 shows the location of the test points on the soft actuator at the top, sides, and bottom. The hardness testing process is carried out to determine the material homogeneity which is carried out on the top side, a bottom side, left the side, and right side of the soft actuator as revealed in Figure 16.
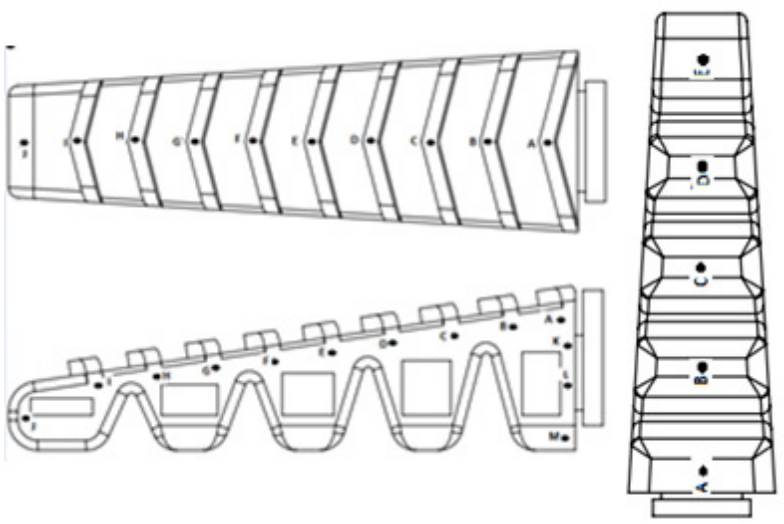

Fig. 15. Test point of the soft actuator.
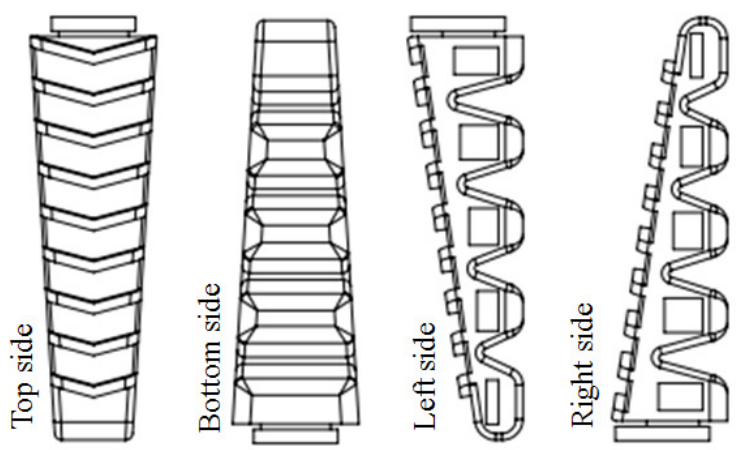

Fig.16. Hardness test section. 
TABLE II. HARDNESS TEST RESULTS

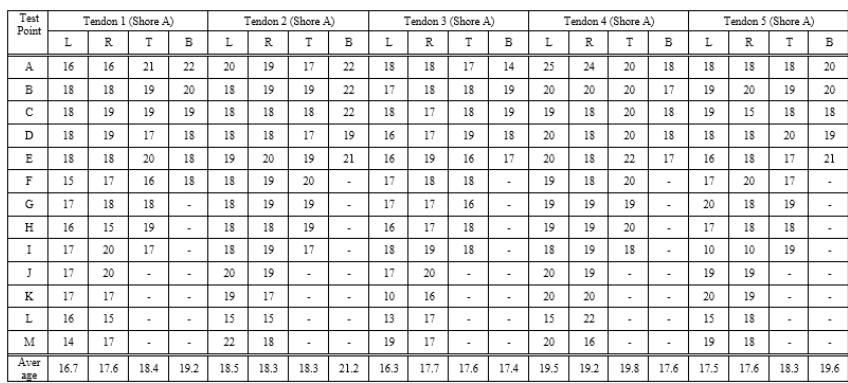

Table II shows the results of the soft actuator test in shore A units which obtained the average results on the left, right, top, and bottom of the tendon. In each part of the actuator, left (L), right (R), top (T) and bottom (B), tendon 1 has an average hardness of $16.7 ; 17.6 ; 18.4 ; 19.2$ shore A. Tendon 2 has an average hardness of $18.5 ; 18.3 ; 18.3 ; 23.2$ shore A. Tendon 3 has an average hardness of $16.3 ; 17.7 ; 17.6 ; 17.4$ shore A. Tendon 4 has an average hardness of $19.5 ; 19.3$; $18.9 ; 17.6$ shore $\mathrm{A}$, and tendon 5 has an average hardness of $17.3 ; 17.4 ; 18.3 ; 19.6$ shore A. Based on actuator hardness test, the smallest level of difference in hardness is tendon 3 . The difference in the value of hardness at each point is caused by several factors, including the level of homogeneity of the mixture of material (silicone rubber and catalyst) which is not evenly mixed. In the next study will use a mixer to mix the constituent material so that the material is homogeneous.

\section{CONCLUSION}

This paper has conveyed how to develop soft motortendon actuators as an alternative soft actuator for a robot that has irregular working trajectories. Five soft actuators designed for soft starfish-like robot have been produced and tested with variations in dimensions and material composition. Based on the bending test, the pitch between the tendon segments and the length of the tendon becomes the most influential factor in determining the ability of the maximum bending angle formed. The greater the value of the dimensions of the two parameters, the greater the angle formed during the bending test, where the third tendon gives the value of the smallest angle error. The soft tendon actuator test without load and without tensile force, tendon 1 has the highest value and the lowest is tendon 3 . This is influenced by the thickness of the tendon. For the testing pot life and curing time, the composition of the material of the soft actuator tendon was created with three variations in composition. Each composition of silicon rubber with a catalyst is equal to $35: 1 ; 25: 1$; and $20: 1$. With the comparison of these compositions, it is known that the pot life period of each is 20 minutes, 10 minutes, 5 minutes; while curing time is 10 hours, 8 hours, 6 hours. Based on these results, the safe composition to suit the soft requirements of the actuator tendon is $35: 1$. For the result of the hardness test, the average hardness was obtained between 16-23 shore A.

\section{ACKNOWLEDGMENT}

This research was financially supported by The LPPM of Diponegoro University on the fiscal year 2018 to 2019.

\section{REFERENCES}

11] R. F.Shepherd, F. Ilievski, F.Choi, S. A. Morin, A. A. Stokes, D.A. Mazzeo, X. Chen, X. Wang, and G. M. Whitesides, "Multigait Soft Robot". Proceedings of the National Academy of Sciences, vol.108, no.51, pp. 20400-20403, 2011.

[2] D. Trivedi, C. D. Rahn, W. M. Kier, and I. D.Walker, "Soft Robotics: Biological Inspiration, State of the Art, and Future Research". Applied Bionics and Biomechanics, vol.5, no.3, pp. 99-117, 2008.

[3] M. C. H. Chua and R. C. H. Yeow, "Propulsion-Based Soft Robotic Actuation". Robotics, vol.6, no.4, pp. 34, 2017.

[4] M. T. Tolley, R. F. Shepherd, B. Mosadegh, K. C. Galloway, M. Wehner, M. Karpelson, and G. M. Whitesides, "A Resilient, Untethered Soft Robot", Soft Robotics, vol.1, no.3, pp. 213-223, 2014

[5] F.Gao, H. Deng, and Y. Zhang, "Hybrid Actuator Combining Shape Memory Alloy with DC Motor for Prosthetic Fingers". Sensors and Actuators : Physical, vol. 223, pp. 40-48, 2015.

[6] L. Paez, G. Agarwal, and J. Paik, "Design and Analysis of a Soft Pneumatic Actuator with Origami Shell Reinforcement". Soft Robotics, vol. 3. no.3, pp. 109-119, 2016.

[7] Yi Sun, Yun Seong Song, and J. Paik, "Characterization of Silicone Rubber Based Soft Pneumatic Actuators". 2013 IEEE/RSJ International Conference on Intelligent Robots and Systems, 2013.

[8] M. P. Groover, "Fundamentals of Modern Manufacturing: Materials, Processes and Systems", 4th ed. USA: John Wiley \& Sons, Inc, 2010.

[9] M. Munadi, M. Ariyanto, J. D. Setiawan and M. F. A. Ayubi, "Development of a Low-Cost Quadrupedal Starfish Soft Robot," 2018 5th International Conference on Information Technology, Computer, and Electrical Engineering (ICITACEE), Semarang, 2018, pp. 225229.

[10] C.-C. Ku and M.-X. Wu, "Evaluation of Service Life of Silicone Rubber Molds using Vacuum Casting". The International Journal of Advanced Manufacturing Technology, nol. 90, no.9-12, pp. 37753781, 2016.

[11] S. Shahria, M. Tariquzzaman, Rahman, M. H.Al Amin, M., Rahman., M. A. "Optimization of Molding Sand Composition for Casting Al Alloy". International Journal of Mechanical Engineering and Applications, vol. 5 vol. 3, pp. 155-161, 2017. 